JOURNAL OF THE

AMERICAN MATHEMATICAL SOCIETY

Volume 21, Number 3, July 2008, Pages 797-808

S 0894-0347(08)00599-7

Article electronically published on March 14, 2008

\title{
ON THE CACHAZO-DOUGLAS-SEIBERG-WITTEN CONJECTURE FOR SIMPLE LIE ALGEBRAS
}

\author{
SHRAWAN KUMAR
}

\section{INTRODUCTION}

Let $\mathfrak{g}$ be a finite dimensional simple Lie algebra over the complex numbers $\mathbb{C}$. Consider the exterior algebra $R:=\bigwedge(\mathfrak{g} \oplus \mathfrak{g})$ on two copies of $\mathfrak{g}$. Then, the algebra $R$ is bigraded with the two copies of $\mathfrak{g}$ sitting in bidegrees $(1,0)$ and $(0,1)$, respectively. To distinguish, we will denote the first copy of $\mathfrak{g}$ by $\mathfrak{g}_{1}$ and the second copy of $\mathfrak{g}$ by $\mathfrak{g}_{2}$. Let $R^{p, q}$ denote the subspace of $R$ consisting of elements of bidegree $(p, q)$. Thus,

$$
R^{p, q}=\bigwedge^{p}\left(\mathfrak{g}_{1}\right) \otimes \bigwedge^{q}\left(\mathfrak{g}_{2}\right) \quad \text { and } \quad R=\bigoplus_{p, q \in \mathbb{Z}_{+}} R^{p, q} .
$$

The bigrading, of course, gives rise to a $\mathbb{Z}_{+}$-grading by declaring any element of $R^{p, q}$ to have total degree $p+q$. Set

$$
R^{n}:=\bigoplus_{p+q=n} R^{p, q}
$$

thus $R^{n}$ consists of elements of total degree $n$.

The diagonal adjoint action of $\mathfrak{g}$ gives rise to a $\mathfrak{g}$-algebra structure on $R$ compatible with the bigrading. We isolate three 'standard' copies of the adjoint representation $\mathfrak{g}$ in $R^{2}$. The $\mathfrak{g}$-module map

$$
\partial: \mathfrak{g} \rightarrow \bigwedge^{2}(\mathfrak{g}), \quad x \mapsto \partial x=\sum_{i}\left[x, e_{i}\right] \wedge f_{i},
$$

considered as a map to $\bigwedge^{2}\left(\mathfrak{g}_{1}\right)$, will be denoted by $c_{1}$, and similarly we define

$$
\begin{aligned}
& c_{2}: \mathfrak{g} \rightarrow \bigwedge^{2}\left(\mathfrak{g}_{2}\right) \text { and } \\
& c_{3}: \mathfrak{g} \rightarrow \mathfrak{g}_{1} \otimes \mathfrak{g}_{2}, \quad x \mapsto \sum_{i}\left[x, e_{i}\right] \otimes f_{i},
\end{aligned}
$$

where $\left\{e_{i}\right\}_{i \leq i \leq N}$ is any basis of $\mathfrak{g}$ and $\left\{f_{i}\right\}_{1 \leq i \leq N}$ is the dual basis of $\mathfrak{g}$ with respect to the normalized Killing form $\langle$,$\rangle of \mathfrak{g}$ (normalized as below). It is easy to see that the three embeddings $c_{d}$ do not depend upon the choice of the basis $\left\{e_{i}\right\}$. We

Received by the editors March 15, 2006.

2000 Mathematics Subject Classification. Primary 22E70, 22E67.

Key words and phrases. Simple Lie algebra, infinite Grassmannian, Abelian ideal.

(C)2008 American Mathematical Society Reverts to public domain 28 years from publication 
fix a Cartan subalgebra $\mathfrak{h}$ of $\mathfrak{g}$ and a Borel subalgebra $\mathfrak{b} \supset \mathfrak{h}$, and normalize the Killing form by demanding that $\langle\theta, \theta\rangle=2$, for the highest root $\theta \in \mathfrak{h}^{*}$. We denote by $C_{d}$ the image of $c_{d}$.

Let $J$ be the (bigraded) ideal of $R$ generated by the three copies $C_{1}, C_{2}, C_{3}$ of $\mathfrak{g}$ (in $R^{2}$ ) and define the bigraded $\mathfrak{g}$-algebra

$$
A:=R / J
$$

The Killing form gives rise to a $\mathfrak{g}$-invariant $S \in A^{1,1}$ given by

$$
S:=\sum_{i} e_{i} \otimes f_{i} .
$$

Motivated by supersymmetric gauge theory, Cachazo-Douglas-Seiberg-Witten CDSW made the following conjecture. They proved the conjecture for $\mathfrak{g}=s l_{N}$ in CDSW, and Witten proved it for $\mathfrak{g}=s p_{N}$ in [W]. He also proved parts (i) and (ii) of the conjecture for $\mathfrak{g}=s o_{N}$ in [W]. Subsequently, Etingof-Kac [EK] (see also E] ) proved the conjecture for $\mathfrak{g}$ of type $G_{2}$ by using the theory of abelian ideals in $\mathfrak{b}$.

Recall that the dual Coxeter number $h$ of $\mathfrak{g}$ is, by definition, $\left\langle\rho, \theta^{\vee}\right\rangle+1$, where $\rho$ is half the sum of all positive roots and $\theta^{\vee}$ is the coroot corresponding to the highest root $\theta$. The value of $h$ for any simple $\mathfrak{g}$ is given as follows (in the bracket): $A_{\ell}(\ell+1) ; B_{\ell}(2 \ell-1) ; C_{\ell}(\ell+1) ; D_{\ell}(2 \ell-2) ; E_{6}(12) ; E_{7}(18) ; E_{8}(30) ; G_{2}(4) ;$ and $F_{4}(9)$.

1.1. Conjecture (CDSW]). (i) The subalgebra $A^{\mathfrak{g}}$ of $\mathfrak{g}$-invariants in $A$ is generated, as an algebra, by the element $S$.

(ii) $S^{h}=0$.

(iii) $S^{h-1} \neq 0$.

Thus, as an algebra,

$$
A^{\mathfrak{g}} \simeq \mathbb{C}[S] /\left\langle S^{h}\right\rangle,
$$

where $\left\langle S^{h}\right\rangle$ denotes the ideal of the polynomial ring $\mathbb{C}[S]$ generated by $S^{h}$.

The aim of this paper is to give a uniform proof of part (i) of the above conjecture. In addition, we give a conjecture (cf. Section 3), the validity of which would imply part (ii) of the above conjecture. For part (iii) of the above conjecture, see Remark 3.2 ,

The proof of part (i) follows from our Theorem 2.2, which is the main result of this paper. This theorem identifies the graded algebra $B^{\mathfrak{g}}$ with the singular cohomology of a certain (finite dimensional) projective subvariety $\mathcal{Y}_{2}$ of the infinite Grassmannian $\mathcal{Y}$ associated to $\mathfrak{g}$, where $B:=R /\left\langle C_{1} \oplus C_{2}\right\rangle$. The definition of the subvariety $\mathcal{Y}_{2}$ is motivated from the theory of abelian ideals in the Borel subalgebra $\mathfrak{b}$ of $\mathfrak{g}$. This theorem is proved by using Garland's result on the Lie algebra cohomology of $\hat{\mathfrak{u}}:=\mathfrak{g} \otimes t \mathbb{C}[t]$, Kostant's result on the 'diagonal' cohomolgy of $\hat{\mathfrak{u}}$ and its connection with abelian ideals in $\mathfrak{b}$, and a certain deformation of the singular cohomology of $\mathcal{Y}$ introduced by Belkale-Kumar [BK].

To obtain part (i) of the above conjecture, observe that the singular cohomology $H^{*}(\mathcal{Y})$ surjects onto $H^{*}\left(\mathcal{Y}_{2}\right)$ under the restriction map. Moreover, as is well known, $H^{*}(\mathcal{Y})$ (being isomorphic with the cohomology of the based loop space $\Omega_{e}(K)$ of a maximal compact subgroup $K$ of $G$ ) is isomorphic with the polynomial ring $\mathbb{C}\left[x_{1}, \ldots, x_{\ell}\right]$ in $\ell$ variables, where $G$ is the connected, simply-connected complex algebraic group with Lie algebra $\mathfrak{g}$ and $\ell$ is its rank. Thus, by virtue of our Theorem 2.2 , we get a surjective algebra homomorphism from $\mathbb{C}\left[x_{1}, \ldots, x_{\ell}\right]$ onto $H^{*}\left(\mathcal{Y}_{2}\right) \simeq$ 
$B^{\mathfrak{g}}$. Finally, we show that each $x_{p}$ (for $p>1$ ) goes to zero under the canonical quotient map $B^{\mathfrak{g}} \rightarrow A^{\mathfrak{g}}$. This is proved by using the explicit expression of the suspension maps associated to the universal principal $K$-bundle $K \rightarrow E(K) \rightarrow$ $B(K)$ and the fibration $\Omega_{e}(K) \rightarrow P_{e}(K) \rightarrow K$, where $P_{e}(K)$ denotes the space of continuous maps $\beta: I \rightarrow K$ from the closed unit interval $I$ starting at $e$. Moreover, it is easy to see that $x_{1}$ goes to the element $S$ (up to a nonzero scalar multiple). This proves part (i) of the above conjecture.

Acknowledgments. It is my pleasure to thank P. Etingof for bringing to my attention the above conjecture and explaining to me his work with Kac. I also thank B. Kostant and R. Suter for some helpful correspondences and the referee for helpful suggestions regarding the exposition. This work was partially supported by NSF grant DMS 0401084.

\section{A tOpOlOGicAl MOdEL FOR $\left(\frac{R}{\left\langle C_{1} \oplus C_{2}\right\rangle}\right)^{\mathfrak{g}}$}

2.1. Notation and preliminaries. Let $G$ be the connected, simply-connected complex algebraic group with Lie algebra $\mathfrak{g}$ and let $\mathcal{G}$ by the corresponding affine Kac-Moody group, which is, by definition, a $\mathbb{C}^{*}$-central extension of the loop group $L(G):=G\left(\mathbb{C}[[t]]\left[t^{-1}\right]\right)$, where $G\left(\mathbb{C}[[t]]\left[t^{-1}\right]\right)$ denotes the $\mathbb{C}[[t]]\left[t^{-1}\right]$-rational points of the algebraic group $G$ (cf. [K2, Section 13.2]). Let $\wp$ be the standard maximal parabolic subgroup, which is, by definition, the $\mathbb{C}^{*}$-extension of $G(\mathbb{C}[[t]])$. Then, the infinite Grassmannian $\mathcal{Y}$ is, by definition,

$$
\mathcal{Y}:=\mathcal{G} / \wp
$$

this is equipped with a projective ind-variety structure. The ring homomorphism $e: \mathbb{C}[[t]] \rightarrow \mathbb{C}, Q \mapsto Q(0)$, gives rise to a group homomorphism $\hat{e}: \wp \rightarrow G$. Let $\mathcal{B}$ be the standard Borel subgroup of $\mathcal{G}$ defined as $\hat{e}^{-1}(B), B$ being the Borel subgroup of $G$ with Lie algebra $\mathfrak{b}$. By the Bruhat decomposition (cf. [K2, §13.2.12]), $\mathcal{Y}$ is the disjoint union of the Bruhat cells

$$
\mathcal{Y}=\bigsqcup_{w \in \operatorname{Aff}(W) / W} \mathcal{B} w \wp / \wp
$$

where $W$ is the Weyl group of $G$ and $\operatorname{Aff}(W)$ is the corresponding affine Weyl group, which is, by definition, the semidirect product of $W$ with the coroot lattice $Q^{\vee} \subset \mathfrak{h}$ acted on via the natural action of $W$ on $\mathfrak{h}$. Aff $(W)$ acts on $\mathfrak{h}$ via the standard action of $W$ on $\mathfrak{h}$, and $Q^{\vee}$ acts on $\mathfrak{h}$ via translation.

Let $\operatorname{Aff}^{\prime}(W) \subset \operatorname{Aff}(W)$ be the set of minimal coset representatives of the $W$ cosets $\operatorname{Aff}(W) / W$. Define the Weyl chamber

$$
\mathfrak{h}_{+}:=\left\{h \in \mathfrak{h}: \alpha_{i}(h) \in \mathbb{R}_{+} \text {for all simple roots } \alpha_{i}\right\} .
$$

Let $C:=\left\{h \in \mathfrak{h}_{+}: \theta(h) \leq 1\right\}$ be the fundamental alcove. Then, $C$ is a fundamental domain for the action of $\operatorname{Aff}(W)$ on $\mathfrak{h}$.

Moreover, as is well known,

$$
\operatorname{Aff}^{\prime}(W)=\left\{w \in \operatorname{Aff}(W): w^{-1} C \subset \mathfrak{h}_{+}\right\} .
$$

We define a closed (finite dimensional) subvariety $\mathcal{Y}_{2}$ of $\mathcal{Y}$ by

$$
\mathcal{Y}_{2}:=\bigcup_{w \in \operatorname{Aff}_{2}^{\prime}(W)} \mathcal{B} w \wp / \wp
$$


where

$$
\operatorname{Aff}_{2}^{\prime}(W):=\left\{w \in \operatorname{Aff}(W): w^{-1} C \subset 2 C\right\} .
$$

Let $\ell$ be the rank of $\mathfrak{g}$, i.e., the dimension of the Cartan subalgebra $\mathfrak{h}$. Since volume $(2 C)=2^{\ell}$ volume $(C)$,

$$
\# \operatorname{Aff}_{2}^{\prime}(W)=2^{\ell} \text {. }
$$

In general, $\mathcal{Y}_{2}$ is not an irreducible variety.

For any topological space $X$, we denote by $H^{*}(X)$ the singular cohomology of $X$ with complex coefficients.

Following is the main result of this paper.

2.2. Theorem. The singular cohomology $H^{*}\left(\mathcal{Y}_{2}\right)$ of $\mathcal{Y}_{2}$ is isomorphic as a $\mathbb{Z}_{+}$graded algebra with the graded algebra $B^{\mathfrak{g}}$, where $B:=R /\left\langle C_{1} \oplus C_{2}\right\rangle$ and $\left\langle C_{1} \oplus C_{2}\right\rangle$ denotes the ideal of $R$ generated by $C_{1} \oplus C_{2}$.

Before we come to the proof of the above theorem, we need to define a new product $\odot_{0}$ in the singular cohomology $H^{*}(\mathcal{Y})$ of $\mathcal{Y}$ as follows. (This was introduced in BK for any flag variety of any semisimple group $\mathcal{H}$ in an attempt to solve the 'eigenvalue problem' for $\mathcal{H}$.)

Let $\left\{\varepsilon^{u}\right\}_{u \in \mathrm{Aff}^{\prime}(W)}$ be the Schubert basis of $H^{*}(\mathcal{Y})$ defined by $\varepsilon^{u}\left(y_{v}\right)=\delta_{u, v}$, for any $v \in \operatorname{Aff}^{\prime}(W)$, where $y_{v}$ is the image in the homology $H_{*}(\mathcal{Y})$ of the fundamental class of the closure $\overline{\mathcal{B} v \wp / \wp}$. Express the usual cup product

$$
\varepsilon^{u} \cdot \varepsilon^{v}=\sum_{w} c_{u, v}^{w} \varepsilon^{w} .
$$

Let $\hat{\mathfrak{g}}$ be the affine Kac-Moody Lie algebra associated to $\mathfrak{g}$. Recall that

$$
\hat{\mathfrak{g}}:=\mathfrak{g} \otimes \mathbb{C}\left[t, t^{-1}\right] \oplus \mathbb{C} c \oplus \mathbb{C} d,
$$

with the bracket defined by

$$
\begin{aligned}
& {\left[t^{m} \otimes x+\lambda c+\mu d, t^{m^{\prime}} \otimes x^{\prime}+\lambda^{\prime} c+\mu^{\prime} d\right]} \\
& \quad=\left(t^{m+m^{\prime}} \otimes\left[x, x^{\prime}\right]+\mu m^{\prime} t^{m^{\prime}} \otimes x^{\prime}-\mu^{\prime} m t^{m} \otimes x\right) \oplus m \delta_{m,-m^{\prime}}\left\langle x, x^{\prime}\right\rangle c,
\end{aligned}
$$

for $\lambda, \mu, \lambda^{\prime}, \mu^{\prime} \in \mathbb{C}, m, m^{\prime} \in \mathbb{Z}$ and $x, x^{\prime} \in \mathfrak{g}$.

Let $\mathcal{T} \subset \mathcal{G}$ be the standard maximal torus of $\mathcal{G}$ and let $\hat{\mathfrak{h}}$ be its Lie algebra. (Actually we have enlarged $\mathcal{G}$ to include $\exp d$.) Then, $\hat{\mathfrak{h}}=\mathfrak{h} \otimes 1 \oplus \mathbb{C} c \oplus \mathbb{C} d$. Let $\left\{\alpha_{0}, \alpha_{1}, \ldots, \alpha_{\ell}\right\}$ be the simple roots of $\hat{\mathfrak{g}}$ such that $\left\{\alpha_{1}, \ldots, \alpha_{\ell}\right\}$ are the simple roots of $\mathfrak{g}$. Thus, $\alpha_{i} \in(\hat{\mathfrak{h}})^{*}$. Choose an element $x_{j} \in \hat{\mathfrak{h}}, 0 \leq j \leq \ell$, satisfying $\alpha_{i}\left(x_{j}\right)=\delta_{i, j}$, for all $0 \leq i \leq \ell$. Also, let $\hat{\rho} \in(\hat{\mathfrak{h}})^{*}$ be an element satisfying $\hat{\rho}\left(\alpha_{i}^{\vee}\right)=1$ for all simple coroots $\alpha_{i}^{\vee}, 0 \leq i \leq \ell$. Now, the new product $\odot_{0}$ is defined by

$$
\varepsilon^{u} \odot_{0} \varepsilon^{v}=\sum_{w \in \operatorname{Aff}^{\prime}(W)} c_{u, v}^{w} \delta_{d_{u, v}^{w}, 0} \varepsilon^{w},
$$

where $\delta$ is the Kronecker delta and

$$
d_{u, v}^{w}:=\left(u^{-1} \hat{\rho}+v^{-1} \hat{\rho}-w^{-1} \hat{\rho}-\hat{\rho}\right)\left(x_{0}\right) .
$$

As proved in [BK, Proposition 17] in the semisimple case, this product is associative (and of course commutative). The extension of this to the present affine Kac-Moody case requires a slight modification of the proof in loc cit.

In fact, one can define this new product $\odot_{0}$ in the cohomology of $\mathcal{H} / \mathcal{I}$ for any symmetrizable Kac-Moody group $\mathcal{H}$ and any standard parabolic subgroup $\mathcal{I}$ of 
finite type and for the cohomology with integral coefficients $\mathbb{Z}$. However, we need this product only for $\mathcal{Y}$. Moreover, there is a $q$-deformation of the cup product in $H^{*}(\mathcal{H} / \mathcal{I}, \mathbb{Z})$ such that the usual cup product corresponds to the value $q=1$, whereas the new product $\odot_{0}$ corresponds to the value $q=0$ (cf. [BK, $\left.\S 6\right]$ ).

For any left $\mathcal{B}$-stable closed subset $\mathcal{Z} \subset \mathcal{Y}$, the kernel of the restriction map $\gamma: H^{*}(\mathcal{Y}) \rightarrow H^{*}(\mathcal{Z})$ is an ideal under the product $\odot_{0}$ in $H^{*}(\mathcal{Y})$. To see this, let $\mathcal{Z}=\bigcup \mathcal{B} w \wp$, where $w$ runs over a certain subset $S_{\mathcal{Z}}$ of $\operatorname{Aff}^{\prime}(W)$. Then,

$$
\operatorname{Ker} \gamma=\bigoplus_{w \in \operatorname{Aff}^{\prime}(W) \backslash S_{\mathcal{Z}}} \mathbb{C} \varepsilon^{w} .
$$

Of course, $\operatorname{Ker} \gamma$ is an ideal of $H^{*}(\mathcal{Y})$ under the cup product. From this and $(*)$, it follows easily that $\operatorname{Ker} \gamma$ is an ideal of $H^{*}(\mathcal{Y})$ under $\odot_{0}$.

This allows us to define the product $\odot_{0}$ in $H^{*}(\mathcal{Z})$, making the restriction map $\gamma: H^{*}(\mathcal{Y}) \rightarrow H^{*}(\mathcal{Z})$ a ring homomorphism under $\odot_{0}$. In particular, we have the product $\odot_{0}$ in $H^{*}\left(\mathcal{Y}_{2}\right)$.

2.3. Lemma. The product $\odot_{0}$ in $H^{*}\left(\mathcal{Y}_{2}\right)$ coincides with the cup product.

Proof. From the definition of $\odot_{0}$, to prove the lemma, it suffices to show that for any $u, v, w \in \operatorname{Aff}_{2}^{\prime}(W)$ such that $c_{u, v}^{w} \neq 0$ in the decomposition (1) following Theorem 2.2 , we have

$$
\left(u^{-1} \hat{\rho}+v^{-1} \hat{\rho}-w^{-1} \hat{\rho}-\hat{\rho}\right)\left(x_{0}\right)=0 .
$$

Since $c_{u, v}^{w} \neq 0$, we, in particular, have

$$
\ell(w)=\ell(u)+\ell(v) .
$$

By [Ko2, Theorems 3.13 and 4.5 and Identity 3.18], for any $u \in \operatorname{Aff}_{2}^{\prime}(W)$,

$$
\hat{\rho}-u^{-1} \hat{\rho}=\ell(u) \delta+\lambda^{u},
$$

for some $\lambda^{u}$ in the root lattice of $\mathfrak{g}$, where $\delta$ is the basic imaginary root of the affine Lie algebra $\hat{\mathfrak{g}}$.

Thus, for any such $u$,

$$
\left(\hat{\rho}-u^{-1} \hat{\rho}\right)\left(x_{0}\right)=\ell(u) .
$$

Combining (2) and (4), we get (1). This proves the lemma.

We now come to the proof of Theorem 2.2.

2.4. Proof of Theorem 2.2. Define the following Lie subalgebras of $\hat{\mathfrak{g}}$ :

$$
\hat{\mathfrak{u}}:=\mathfrak{g} \otimes t \mathbb{C}[t], \quad \hat{\mathfrak{u}}^{-}:=\mathfrak{g} \otimes t^{-1} \mathbb{C}\left[t^{-1}\right], \quad \mathfrak{g}_{0}:=\mathfrak{g} \otimes 1 \oplus \mathbb{C} c \oplus \mathbb{C} d .
$$

By a result of Garland (cf. [K2, Theorem 3.2.7]), as a $\mathfrak{g}_{0}$-module, the Lie algebra cohomology

$$
H^{p}\left(\hat{\mathfrak{u}}^{-}\right) \simeq \bigoplus_{w \in \operatorname{Aff}^{\prime}(W), \ell(w)=p} L_{0}\left(w^{-1} \hat{\rho}-\hat{\rho}\right)^{*},
$$

where $L_{0}(\lambda)$ denotes the irreducible $\mathfrak{g}_{0}$-module with highest weight $\lambda$. Similarly, by [K2, (3.2.11.3)],

$$
H^{p}(\hat{\mathfrak{u}}) \simeq \bigoplus_{w \in \operatorname{Aff}^{\prime}(W), \ell(w)=p} L_{0}\left(w^{-1} \hat{\rho}-\hat{\rho}\right)
$$


By [BK, Theorem 43], there is a graded algebra isomorphism

$$
\phi: H^{*}\left(\mathcal{Y}, \odot_{0}\right) \simeq\left[H^{*}(\hat{\mathfrak{u}}) \otimes H^{*}\left(\hat{\mathfrak{u}}^{-}\right)\right]^{\mathfrak{g}_{0}},
$$

where $H^{*}\left(\mathcal{Y}, \odot_{0}\right)$ denotes $H^{*}(\mathcal{Y})$ equipped with the product $\odot_{0}$. (Even though in [BK] the theorem is proved for semisimple Lie algebras, a similar proof carries over and gives the result for any symmetrizable Kac-Moody case by using results from [K0, §3].) Moreover, under the above identifications (1) and (2), for any $w \in \operatorname{Aff}^{\prime}(W)$,

$$
\phi\left(\varepsilon^{w}\right) \in\left[L_{0}\left(w^{-1} \hat{\rho}-\hat{\rho}\right) \otimes L_{0}\left(w^{-1} \hat{\rho}-\hat{\rho}\right)^{*}\right]^{\mathfrak{g}_{0}} .
$$

The cohomology modules $H^{p}(\hat{\mathfrak{u}})$ and $H^{p}\left(\hat{\mathfrak{u}}^{-}\right)$acquire a grading coming from the total degree of $t$ in $\bigwedge^{p}(\hat{\mathfrak{u}})$ and $\bigwedge^{p}\left(\hat{\mathfrak{u}}^{-}\right)$, respectively. Thus,

$$
H^{p}(\hat{\mathfrak{u}})=\bigoplus_{m \in \mathbb{Z}_{+}} H_{(-m)}^{p}(\hat{\mathfrak{u}}),
$$

where $H_{(-m)}^{p}(\hat{\mathfrak{u}})$ denotes the space of elements of $H^{p}(\hat{\mathfrak{u}})$ of total $t$-degree $-m$. Similarly,

$$
H^{p}\left(\hat{\mathfrak{u}}^{-}\right)=\bigoplus_{m \in \mathbb{Z}_{+}} H_{(m)}^{p}\left(\hat{\mathfrak{u}}^{-}\right)
$$

Clearly,

$$
H_{(-m)}^{p}(\hat{\mathfrak{u}})=H_{(m)}^{p}\left(\hat{\mathfrak{u}}^{-}\right)=0, \quad \text { if } m<p .
$$

Let $H_{D}^{*}(\hat{\mathfrak{u}})$ denote the 'diagonal' cohomology

$$
H_{D}^{*}(\hat{\mathfrak{u}}):=\bigoplus_{p \in \mathbb{Z}_{+}} H_{(-p)}^{p}(\hat{\mathfrak{u}})
$$

and similarly,

$$
H_{D}^{*}\left(\hat{\mathfrak{u}}^{-}\right):=\bigoplus_{p \in \mathbb{Z}_{+}} H_{(p)}^{p}\left(\hat{\mathfrak{u}}^{-}\right) .
$$

Then, clearly, $H_{D}^{*}(\hat{\mathfrak{u}})$ and $H_{D}^{*}\left(\hat{\mathfrak{u}}^{-}\right)$are graded $\mathfrak{g}$-subalgebras of $H^{*}(\hat{\mathfrak{u}})$ and $H^{*}\left(\hat{\mathfrak{u}}^{-}\right)$, respectively. By [Ko2, Theorem 4.16], as graded $\mathfrak{g}$-algebras,

$$
H_{D}^{*}(\hat{\mathfrak{u}}) \simeq \frac{\bigwedge(\mathfrak{g})}{\langle\partial(\mathfrak{g})\rangle}
$$

where $\partial: \mathfrak{g} \rightarrow \bigwedge^{2}(\mathfrak{g})$ is the map defined in Section 1 and $\langle\partial(\mathfrak{g})\rangle$ denotes the ideal of the exterior algebra $\bigwedge(\mathfrak{g})$ generated by $\partial(\mathfrak{g})$. Similarly,

$$
H_{D}^{*}\left(\hat{\mathfrak{u}}^{-}\right) \simeq \frac{\bigwedge(\mathfrak{g})}{\langle\partial(\mathfrak{g})\rangle} .
$$

Moreover, by [Ko0] and [Ko1, the $\mathfrak{g}$-module $\frac{\Lambda(\mathfrak{g})}{\langle\partial(\mathfrak{g})\rangle}$ is a multiplicity-free $\mathfrak{g}$-module. In fact, for any abelian ideal $I \subset \mathfrak{b}$ (which is automatically contained in $\mathfrak{n}:=[\mathfrak{b}, \mathfrak{b}]$ ), consider the line $\bigwedge^{\operatorname{dim} I}(I) \subset \bigwedge^{\operatorname{dim} I}(\mathfrak{g})$. Then, $I$ being an ideal in $\mathfrak{b}, \bigwedge^{\operatorname{dim} I}(I)$ is stable under $\mathfrak{b}$. Let $V_{I} \subset \bigwedge^{\operatorname{dim} I}(\mathfrak{g})$ be the (irreducible) $\mathfrak{g}$-module generated by $\bigwedge^{\operatorname{dim} I}(I)$. Then, as $\mathfrak{g}$-modules, the canonical map (induced by the inclusion)

$$
\bigoplus_{I \in \Xi} V_{I} \rightarrow \frac{\bigwedge(\mathfrak{g})}{\langle\partial(\mathfrak{g})\rangle}
$$


is an isomorphism, where $\Xi$ is the set of all the abelian ideals of $\mathfrak{b}$. By a result of D. Peterson, $\Xi$ has cardinality $2^{\ell}, \ell$ being the rank of $\mathfrak{g}$. Further, for any $p \geq 0$, $\frac{\Lambda^{p}(\mathfrak{g})}{\langle\partial(\mathfrak{g})\rangle \cap \Lambda^{p}(\mathfrak{g})}$ is a self-dual $\mathfrak{g}$-module. Thus, as graded algebras,

$$
\left[H_{D}^{*}(\hat{\mathfrak{u}}) \otimes H_{D}^{*}\left(\hat{\mathfrak{u}}^{-}\right)\right]^{\mathfrak{g}_{0}} \simeq\left[\frac{\bigwedge(\mathfrak{g})}{\langle\partial(\mathfrak{g})\rangle} \otimes \frac{\bigwedge(\mathfrak{g})}{\langle\partial(\mathfrak{g})\rangle}\right]^{\mathfrak{g}}=: B^{\mathfrak{g}} .
$$

We next show that the composite $\xi:=\gamma \circ \phi^{-1} \circ i$,

$$
\begin{aligned}
{\left[H_{D}^{*}(\hat{\mathfrak{u}}) \otimes H_{D}^{*}\left(\hat{\mathfrak{u}}^{-}\right)\right]^{\mathfrak{g}_{0}} } & \stackrel{i}{\hookrightarrow}\left[H^{*}(\hat{\mathfrak{u}}) \otimes H^{*}\left(\hat{\mathfrak{u}}^{-}\right)\right]^{\mathfrak{g}_{0}} \\
& \stackrel{\phi^{-1}}{\longrightarrow} H^{*}\left(\mathcal{Y}, \odot_{0}\right) \stackrel{\gamma}{\longrightarrow} H^{*}\left(\mathcal{Y}_{2}, \odot_{0}\right),
\end{aligned}
$$

is an algebra isomorphism, where $i$ is the inclusion and $\gamma$ is the restriction map.

By (1) of Section 2.1, the total dimension

$$
\operatorname{dim} H^{*}\left(\mathcal{Y}_{2}\right)=2^{\ell} .
$$

Also, by the above discussion,

$$
\operatorname{dim}\left[H_{D}^{*}(\hat{\mathfrak{u}}) \otimes H_{D}^{*}\left(\hat{\mathfrak{u}}^{-}\right)\right]^{\mathfrak{g}_{0}}=2^{\ell} .
$$

Observe that all the maps $i, \phi^{-1}$ and $\gamma$ are algebra homomorphisms. Thus, it suffices to show that $\xi$ is surjective. Before we give a precise proof for the surjectivity of $\xi$, let us briefly outline why the 'diagonal' part of the cohomology suffices. By Kostant's results $(3)$ and $(4)$, the $\mathfrak{g}$-module $H_{D}^{*}(\hat{\mathfrak{u}})$ decomposes as a direct sum of irreducible $\mathfrak{g}$-modules parametrized by $\Xi$. Moreover, by a result of Peterson, $\Xi$ is parametrized by the subset $\operatorname{Aff}_{2}^{\prime}(W)$ of the affine Weyl group. Further, from the definition of $\mathcal{Y}_{2}$, the Schubert basis of the cohomology $H^{*}\left(\mathcal{Y}_{2}\right)$ is also parametrized by $\operatorname{Aff}_{2}^{\prime}(W)$.

We now give a precise proof for the surjectivity of $\xi$. Take $w \in \operatorname{Aff}_{2}^{\prime}(W)$ and consider the one dimensional subspace

$$
V(w):=\left[L_{0}\left(w^{-1} \hat{\rho}-\hat{\rho}\right) \otimes L_{0}\left(w^{-1} \hat{\rho}-\hat{\rho}\right)^{*}\right]^{\mathfrak{g}_{0}} \text { of }\left[H^{*}(\hat{\mathfrak{u}}) \otimes H^{*}\left(\hat{\mathfrak{u}}^{-}\right)\right]^{\mathfrak{g}_{0}}
$$

under the identifications (1) and (2) of this section. Under the isomorphism $\phi^{-1}$,

$$
\phi^{-1}(V(w))=\mathbb{C} \varepsilon^{w} .
$$

On the other hand, by [Ko2, Theorem 4.5 and Remark 4.13], for $w \in \operatorname{Aff}_{2}^{\prime}(W)$, $V(w) \subset \operatorname{Im}(i)$. This proves the surjectivity of $\xi$ and hence concludes the proof that $\xi$ is a graded algebra isomorphism. Combining this with the identification (5) and Lemma 2.3, we get Theorem 2.2.

By a result of Garland-Raghunathan GR and Quillen (unpublished), the space $\mathcal{Y}$ is homotopically equivalent to the continuous based loop group $\Omega_{e}(K)$ of a maximal compact subgroup $K$ of $G$. In particular,

$$
H^{*}(\mathcal{Y}) \simeq H^{*}\left(\Omega_{e}(K)\right) .
$$


2.5. The generators of $H^{*}\left(\boldsymbol{\Omega}_{e}(K)\right)$. Let $F$ be the ring $\mathbb{C}\left[t, t^{-1}\right]$ of Laurent polynomials. For any invariant homogeneous polynomial $P \in S\left(\mathfrak{g}^{*}\right)^{\mathfrak{g}}$ of degree $d+1$ $(d \geq 1)$, define the map

$$
\widetilde{\phi}_{P}: \bigwedge_{\mathbb{C}}^{2 d}(\mathfrak{g} \otimes F) \rightarrow \Omega_{F}^{1}
$$

by

$$
\begin{aligned}
\widetilde{\phi}_{P}\left(v_{0}\right. & \left.\wedge v_{1} \wedge \cdots \wedge v_{2 d-1}\right) \\
& =\sum_{\sigma \in S_{2 d}} \varepsilon(\sigma) P\left(v_{\sigma(0)},\left[v_{\sigma(1)}, v_{\sigma(2)}\right], \ldots,\left[v_{\sigma(2 d-3)}, v_{\sigma(2 d-2)}\right], d v_{\sigma(2 d-1)}\right),
\end{aligned}
$$

for $v_{i} \in \mathfrak{g} \otimes F$, where the loop algebra $\mathfrak{g} \otimes F$ has the standard bracket, $\Omega_{F}^{1}$ is the space of algebraic 1-forms on the affine variety $\mathbb{C}^{*}, d(x \otimes a(t)):=x \otimes \frac{d a(t)}{d t} d t \in \mathfrak{g} \otimes \Omega_{F}^{1}$ for $x \in \mathfrak{g}$ and $a(t) \in F$, and $P$ is extended $F$-linearly. Define the residue map $\alpha: \Omega_{F}^{1} \rightarrow \mathbb{C}$ by

$$
\alpha(Q(t) d t)=\text { the coefficient of } \frac{1}{t} \text { in } Q(t) .
$$

Of course, $\alpha(Q(t) d t)=\frac{1}{2 \pi i} \int_{S^{1}} Q(t) d t$. Composing $\widetilde{\phi}_{P}$ with $\alpha$, we get the map

$$
\phi_{P}: \bigwedge_{\mathbb{C}}^{2 d}(\mathfrak{g} \otimes F) \rightarrow \mathbb{C} .
$$

Then, the restriction of $\phi_{P}$ to $\bigwedge_{\mathbb{C}}^{2 d}\left(L^{0}(\mathfrak{g})\right)$ represents a relative cocycle for the Lie algebra pair $(\mathfrak{g} \otimes F, \mathfrak{g})$ (where $L^{0}(\mathfrak{g})$ is the kernel of $\left.\mathfrak{g} \otimes F \rightarrow \mathfrak{g}, x \otimes Q \mapsto Q(1) x\right)$, thus giving rise to a Lie algebra cohomology class $\left[\phi_{P}\right] \in H^{2 d}(\mathfrak{g} \otimes F, \mathfrak{g})$.

We recall the following result from [K1, Theorem 1.6 and Lemma 1.8].

2.6. Theorem. The integration map (appropriately defined) defines an algebra isomorphism in cohomology:

$$
H^{*}(\mathfrak{g} \otimes F, \mathfrak{g}) \simeq H^{*}(\mathcal{Y}) \simeq H^{*}\left(\Omega_{e}(K)\right) .
$$

From now on, we will identify $H^{*}(\mathfrak{g} \otimes F, \mathfrak{g})$ with $H^{*}(\mathcal{Y})$.

As is well known, $S\left(\mathfrak{g}^{*}\right)^{\mathfrak{g}}$ is freely generated (as a commutative algebra) by certain homogeneous polynomials $P_{1}, \ldots, P_{\ell}$ of degrees $m_{1}+1, m_{2}+1, \ldots, m_{\ell}+1$, respectively, where $m_{1}=1<m_{2} \leq \cdots \leq m_{\ell}$ are the exponents of $\mathfrak{g}$. Moreover, $H^{*}\left(\Omega_{e}(K)\right)$ is freely generated (again as a commutative algebra) by taking the double suspension $\hat{P}_{1}, \ldots, \hat{P}_{\ell}$ of the elements $P_{1}, \ldots, P_{\ell}$, respectively; first associated to the universal principal $K$-bundle $K \rightarrow E(K) \rightarrow B(K)$ and then to the fibration $\Omega_{e}(K) \rightarrow P_{e}(K) \rightarrow K$, where $P_{e}(K)$ denotes the space of continuous maps $\beta: I \rightarrow K$ from the closed unit interval $I$ starting at $e$ (i.e., $\beta(0)=e$ ) (cf. B, Proposition 7.1]). (Here we have identified $H^{*}(B(K)) \simeq S\left(\mathfrak{g}^{*}\right)^{\mathfrak{g}}$.) Observe that $\hat{P}_{p} \in H^{2 m_{p}}\left(\Omega_{e}(K)\right)$.

Then, the class $\hat{P}_{p}$ corresponds to the class $\left[\phi_{P_{p}}\right] \in H^{2 m_{p}}(\mathfrak{g} \otimes F, \mathfrak{g})$ under the identification of Theorem 2.6 (cf. [CS, Proposition 3.2], where an explicit description of the first suspension is given, combined with [B, $\S 7]$, where the second suspension is described explicitly; alternatively see [PS, Proposition (4.11.3)]).

Combining this with Theorem 2.6, we get the following.

2.7. Theorem. The cohomology classes $\left[\phi_{P_{1}}\right], \ldots,\left[\phi_{P_{\ell}}\right] \in H^{*}(\mathfrak{g} \otimes F, \mathfrak{g})$ (with degrees $2 m_{1}, \ldots, 2 m_{\ell}$, respectively) generate the cohomology $H^{*}(\mathfrak{g} \otimes F, \mathfrak{g}$ ) freely (as a commutative algebra). 
Theorems 2.2 and 2.7 readily prove the first part of Conjecture 1.1. Specifically, we have the following.

2.8. Theorem. With the notation as in Section 1, the algebra $A^{\mathfrak{g}}$ is generated, as an algebra, by the element $S$.

Proof. By Theorem 2.7, the polynomial ring

$$
\mathbb{C}\left[x_{1}, \ldots, x_{\ell}\right] \stackrel{\sim}{\longrightarrow} H^{*}(\mathcal{Y}), x_{p} \mapsto\left[\phi_{P_{p}}\right] .
$$

Observe next that the restriction map $\gamma: H^{*}(\mathcal{Y}) \rightarrow H^{*}\left(\mathcal{Y}_{2}\right)$ is surjective. This follows since, by the Bruhat decomposition of $\mathcal{Y}$ as in Section 2.1, $\mathcal{Y}$ is a $\mathrm{CW}$ complex obtained from $\mathcal{Y}_{2}$ by attaching (real) even dimensional cells. In particular, composing the above isomorphism with $\gamma$, we get a surjection

$$
\eta: \mathbb{C}\left[x_{1}, \ldots, x_{\ell}\right] \rightarrow H^{*}\left(\mathcal{Y}_{2}\right) .
$$

We next show that, under the identification of $H^{*}\left(\mathcal{Y}_{2}\right)$ with the algebra $B^{\mathfrak{g}}$ (guaranteed by Theorem 2.2), the element $x_{1} \mapsto z S$ (for a nonzero $z \in \mathbb{C}$ ) and, moreover, each $\eta\left(x_{p}\right), p>1$, lies in the kernel of the standard quotient map $B^{\mathfrak{g}} \rightarrow$ $A^{\mathfrak{g}}$.

We first prove the assertion that $\eta\left(x_{1}\right)=z S$. Since $H^{2}\left(\mathcal{Y}_{2}\right)$ is one dimensional and $\eta\left(x_{p}\right)$ is a cohomology class of degree $2 m_{p}>2$ for any $p>1, \eta\left(x_{1}\right)$ is the unique nonzero element of $H^{2}\left(\mathcal{Y}_{2}\right)$ (up to a nonzero scalar multiple). Further, by Theorem 2.2,

$$
H^{2}\left(\mathcal{Y}_{2}\right) \simeq\left(B^{2}\right)^{\mathfrak{g}},
$$

where $B^{2}$ is the total degree 2 component of $B$. But, $S$ is the unique element of $\left(B^{2}\right)^{\mathfrak{g}}$ (up to scalar multiples). This proves the assertion that $\eta\left(x_{1}\right)=z S$.

We next prove that $\eta\left(x_{p}\right)$, for $p>1$, lies in the kernel of $B^{\mathfrak{g}} \rightarrow A^{\mathfrak{g}}$. The computation is easy at its core; the appearance of brackets in all the generators beyond $x_{1}$ places them in the kernel of the projection $B^{\mathfrak{g}} \rightarrow A^{\mathfrak{g}}$. We now give a precise and detailed proof.

Take dual bases $\left\{e_{i}\right\}$ and $\left\{f_{i}\right\}$ of $\mathfrak{g}$ as in Section 1. Then, $\left\{e_{i}(n):=e_{i} \otimes t^{n}\right\}_{n \in \mathbb{Z}}$ forms a basis of $\mathfrak{g} \otimes F$. Identify $\left(\mathfrak{g} \otimes t^{n}\right)^{*}$ with $\mathfrak{g} \otimes t^{n}$ as a $\mathfrak{g}$-module via the Killing form on the $\mathfrak{g}$-factor. Then, $\left\{f_{i}(n):=f_{i} \otimes t^{n}\right\}_{n \in \mathbb{Z}}$ is the dual basis of

$$
(\mathfrak{g} \otimes F)^{\vee}:=\bigoplus_{n \in \mathbb{Z}}\left(\mathfrak{g} \otimes t^{n}\right)^{*} \simeq \mathfrak{g} \otimes F .
$$

For any $\sigma \in S_{2 d}$ and $P \in S^{d+1}\left(\mathfrak{g}^{*}\right)^{\mathfrak{g}}(d \geq 2)$, consider the linear form

$$
\phi_{P, \sigma}: \bigotimes_{\mathbb{C}}^{2 d}(\mathfrak{g} \otimes F) \rightarrow \mathbb{C}
$$

defined by

$$
\begin{aligned}
\phi_{P, \sigma}\left(v_{0} \otimes v_{1}\right. & \left.\otimes \cdots \otimes v_{2 d-1}\right) \\
& =\alpha\left(P\left(v_{\sigma(0)},\left[v_{\sigma(1)}, v_{\sigma(2)}\right], \ldots,\left[v_{\sigma(2 d-3)}, v_{\sigma(2 d-2)}\right], d v_{\sigma(2 d-1)}\right)\right),
\end{aligned}
$$

where $\alpha: \Omega_{F}^{1} \rightarrow \mathbb{C}$ is the map defined in Section 2.5. For notational convenience, assume $\sigma(1)<\sigma(2)$. For any fixed

$$
v_{0}, v_{1}, \ldots, \hat{v}_{\sigma(1)}, \ldots, \hat{v}_{\sigma(2)}, \ldots, v_{2 d-1} \in \mathfrak{g} \otimes F,
$$


consider the restriction $\bar{\phi}_{P, \sigma}$ of the function $\phi_{P, \sigma}$ to

$$
v_{0} \times v_{1} \times \cdots \times \mathfrak{g} \otimes F \times \cdots \times \mathfrak{g} \otimes F \times \cdots \times v_{2 d-1},
$$

where the two copies of $\mathfrak{g} \otimes F$ are placed in the $\sigma(1)$ and $\sigma(2)$ slots. Then, under the above identification (1),

$$
\begin{aligned}
\bar{\phi}_{P, \sigma} & =\sum_{i, j, m, n} f_{i}(n) \otimes f_{j}(m) \alpha\left(P \left(v_{\sigma(0)},\left[e_{i}(n), e_{j}(m)\right],\left[v_{\sigma(3)}, v_{\sigma(4)}\right], \ldots,\right.\right. \\
& {\left.\left.\left[v_{\sigma(2 d-3)}, v_{\sigma(2 d-2)}\right], d v_{\sigma(2 d-1)}\right)\right) } \\
& =\sum_{i, j, m, n, k} f_{i}(n) \otimes f_{j}(m) \alpha\left(P\left(-,\left\langle\left[e_{i}, e_{j}\right], e_{k}\right\rangle f_{k}(n+m),-\right)\right) \\
& =\sum_{i, j, m, n, k}\left\langle e_{i},\left[e_{j}, e_{k}\right]\right\rangle f_{i}(n) \otimes f_{j}(m) \alpha\left(P\left(-, f_{k}(n+m),-\right)\right) \\
& =\sum_{j, k, m, n}\left[e_{j}, e_{k}\right](n) \otimes f_{j}(m) \alpha\left(P\left(-, f_{k}(n+m),-\right)\right) \\
& =-\sum_{j, k, m, n}\left[e_{k}, e_{j}\right](n) \otimes f_{j}(m) \alpha\left(P\left(-, f_{k}(n+m),-\right)\right) .
\end{aligned}
$$

Recall the definition of the isomorphism

$$
\xi:\left[H_{D}^{*}(\hat{\mathfrak{u}}) \otimes H_{D}^{*}\left(\hat{\mathfrak{u}}^{-}\right)\right]^{\mathfrak{g}_{0}} \rightarrow H^{*}\left(\mathcal{Y}_{2}\right)
$$

from Section 2.4. In particular, any class $c \in H^{2 p}\left(\mathcal{Y}_{2}\right)$ is the restriction of a class in $H^{2 p}(\mathcal{Y})$ represented by a relative cocycle $\omega$ of the Lie algebra pair $(\mathfrak{g} \otimes F, \mathfrak{g})$ lying in the linear span of

$$
\left\{f_{i_{1}}(1) \wedge \cdots \wedge f_{i_{p}}(1) \wedge f_{j_{1}}(-1) \wedge \cdots \wedge f_{j_{p}}(-1)\right\}_{\substack{i_{1}<\cdots<i_{p} \\ j_{1}<\cdots<j_{p}}}
$$

(Here, we have identified $(\mathfrak{g} \otimes F)^{\vee}$ with $\mathfrak{g} \otimes F$ as earlier.) Also, recall the isomorphism

$$
\left[H_{D}^{*}(\hat{\mathfrak{u}}) \otimes H_{D}^{*}\left(\hat{\mathfrak{u}}^{-}\right)\right]^{\mathfrak{g}_{0}} \simeq B^{\mathfrak{g}}
$$

from (5) of Section 2.4. These two isomorphisms put together give rise to the identification of Theorem 2.2:

$$
H^{*}\left(\mathcal{Y}_{2}\right) \stackrel{\sim}{\longrightarrow} B^{\mathfrak{g}}
$$

From the definition of the map $\eta: \mathbb{C}\left[x_{1}, \ldots, x_{\ell}\right] \rightarrow H^{*}\left(\mathcal{Y}_{2}\right)$ and $(2)$, we see that, for all $p>1, \eta\left(x_{p}\right)$ belongs to the ideal of $B$ generated by the copy $C_{3}$ of the adjoint representation. To prove this observe that, from the above discussion, in the decomposition $(2)$ of $\bar{\phi}_{P, \sigma}$ we can take $(n, m)$ to be one of $(1,1),(-1,-1),(1,-1)$ or $(-1,1)$. The terms in the right side of $(2)$ of the form $(1,1)$ (resp. $(-1,-1))$ lie in the ideal generated by $C_{1}$ (resp. $C_{2}$ ), whereas the terms of the form $(1,-1)$ and $(-1,1)$ lie in the ideal generated by $C_{3}$. Thus, $\eta\left(x_{p}\right)$ goes to 0 under the quotient map $B^{\mathfrak{g}} \rightarrow A^{\mathfrak{g}}$. This proves the theorem.

\section{A CONJECture}

As in Section 2.4, let $\Xi$ be the set of all the abelian ideals in the Borel subalgebra $\mathfrak{b}$. Let $\Xi^{o}$ be the subset consisting of those abelian ideals $I$ such that for any root space $\mathfrak{g}_{\alpha}$ corresponding to the root $\alpha$, if $\mathfrak{g}_{\alpha} \subset I$, then $\langle\alpha, \theta\rangle \neq 0$, where $\theta$ is the highest root. 
Recall that there is a bijection $\zeta: \Xi \rightarrow \operatorname{Aff}_{2}^{\prime}(W)$ such that for any $I \in \Xi, \operatorname{dim} I=$ $\ell(\zeta(I))$ (see, e.g., Ko2, Theorem 4.4]). Actually, we take $\zeta(I)=\left(\sigma_{I}\right)^{-1}$, where $\sigma$ is the map as in loc cit. Denote the image $\zeta\left(\Xi^{o}\right)$ by $\operatorname{Aff}_{2}^{\prime}(W)^{o}$ and consider the subvariety of $\mathcal{Y}_{2}$ :

$$
\mathcal{Z}:=\bigcup_{w \in \mathrm{Aff}_{2}^{\prime}(W)^{\circ}} \mathcal{B} w \wp / \wp
$$

3.1. Conjecture. Consider the map $\Phi: H^{*}\left(\mathcal{Y}_{2}\right) \rightarrow A^{\mathfrak{g}}$ obtained by the isomorphism $H^{*}\left(\mathcal{Y}_{2}\right) \stackrel{\sim}{\longrightarrow} B^{\mathfrak{g}}$ of Theorem 2.2 followed by the standard projection $B^{\mathfrak{g}} \rightarrow$ $A^{\mathfrak{g}}$. Then, $\Phi$ factors through the cohomology $H^{*}(\mathcal{Z})$ under the restriction map $H^{*}\left(\mathcal{Y}_{2}\right) \rightarrow H^{*}(\mathcal{Z})$.

By Suter [S, Proposition 11], for any $I \in \Xi^{o}, \operatorname{dim} I \leq h-1$. In particular, this gives

$$
\operatorname{dim}_{\mathbb{C}} \mathcal{Z} \leq h-1 .
$$

So, the validity of the above conjecture will readily imply the validity of part (ii) of Conjecture 1.1.

3.2. Remark. Witten has outlined an idea to prove part (iii) of Conjecture 1.1 by using the based one-instanton moduli space associated to $K$ and the flat four dimensional space $\mathbb{R}^{4}$ (cf. [W, Section 5]). We are in the process of developing his ideas into a mathematically rigorous proof, but there are still some technical difficulties to overcome.

\section{REFERENCES}

[BK] P. Belkale and S. Kumar, Eigenvalue problem and a new product in cohomology of flag varieties, Inventiones Math. 166 (2006), 185-228. MR2242637(2007k:14097)

[B] R. Bott, The space of loops on a Lie group, Michigan Math. J. 5 (1958), 35-61. MR0102803 (21:1589)

[CDSW] F. Cachazo, M.R. Douglas, N. Seiberg, and E. Witten, Chiral rings and anomalies in supersymmetric gauge theory, J. High Energy Phys. 12 (2002). MR.1960462 (2003m:81240)

[CS] S.S. Chern and J. Simons, Characteristic forms and geometric invariants, Annals of Math. 99 (1974), 48-69. MR0353327 (50:5811)

[E] P. Etingof, On the Cachazo-Douglas-Seiberg-Witten conjecture for simple Lie algebras, II, Preprint (2004).

[EK] P. Etingof and V. Kac, On the Cachazo-Douglas-Seiberg-Witten conjecture for simple Lie algebras, Preprint (2003).

[GR] H. Garland and M.S. Raghunathan, A Bruhat decomposition for the loop space of a compact group: A new approach to results of Bott, Proc. Natl. Acad. Sci. USA $\mathbf{7 2}$ (1975), 4716-4717. MR0417333(54:5389)

[Ko0] B. Kostant, Eigenvalues of a Laplacian and commutative Lie subalgebras, Topology 3 (1965), 147-159. MR0167567 (29:4839)

[Ko1] B. Kostant, On $\wedge(\mathfrak{g})$ for a semisimple Lie algebra $\mathfrak{g}$, as an equivariant module over the symmetric algebra $S(\mathfrak{g}), A d v$. Stud. Pure Math. 26 (2000), 129-144. MR 1770720 (2001g:17009)

[Ko2] B. Kostant, Powers of the Euler product and commutative subalgebras of a complex simple Lie algebra, Inventiones Math. 158 (2004), 181-226. MR2090363 (2005m:17007)

[K0] S. Kumar, Geometry of Schubert cells and cohomology of Kac-Moody Lie algebras, J. Diff. Geometry 20 (1984), 389-431. MR0788286 (86j:17020)

[K1] S. Kumar, Rational homotopy theory of flag varieties associated to Kac-Moody groups, in: Infinite Dimensional Groups with Applications, MSRI Publications vol. 4, SpringerVerlag (1985), 233-273. MR823322 (87c:17026)

[K2] S. Kumar, Kac-Moody Groups, their Flag Varieties and Representation Theory, Progress in Math. vol. 204, Birkhäuser (2002). MR.1923198(2003k:22022) 
[PS] A. Pressley and G. Segal, Loop Groups, Clarendon Press, Oxford (1992). MR0900587 (88i:22049)

[S] R. Suter, Abelian ideals in a Borel subalgebra of a complex simple Lie algebra, Inventiones Math. 156 (2004), 175-221. MR2047661 (2005b:17020)

[W] E. Witten, Chiral ring of $S p(N)$ and $S O(N)$ supersymmetric gauge theory in four dimensions, Chinese Ann. of Math., Ser. B 24 (2003), 403-414. MR2024979 (2004k:81387)

Department of Mathematics, University of North Carolina, Chapel Hill, North CarOLINA 27599-3250

E-mail address: shrawan@email.unc.edu 